\title{
EXPERIMENTAL STUDY OF THE PLASMA BEAT WAVE ACCELERATOR
}

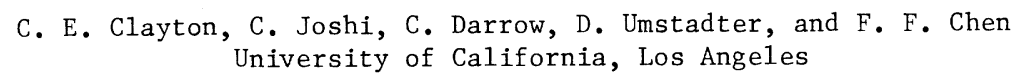

Los Angeles, CA 90024

\section{Abstract}

The physics of the beat-excited, relativistic electron plasma wave, a candidate for a laser particle accelerator, is studied in experiments at UCLA. Preliminary experiments have shown waves excited up to $1-3 \%$ of the cold wavebreaking amplitude, corresponding to longitudinal electric fields of $0.3-$ $1.0 \mathrm{GeV} / \mathrm{m}$. Issues addressed here include possible experimental limitations on the length, growth time, and amplitude of this plasma wave. Plans for future experiments on the acceleration of test charges will also be presented.

\section{Introduction}

Among the proposed schemes for the high gradient acceleration of particles with laser beams are the media accelerators. (1) The medium is used to essentially transform the transverse (to $k_{0}$ ) laser electric field into a longitudinal field which can then be used to accelerate particles. One such media accelerator is the plasma beatwave accelerator. (2) Here, a laser beam containing two frequencies, $\omega_{0}$ and $\omega_{1}$ with $\omega_{0}-\omega_{1}=\Delta \omega$, is injected into a plasma whose electron density $n_{0}$ is such that the plasma frequency $\omega_{p}\left[=\left(4 \pi n_{0} e^{2} / m\right)^{\frac{1}{2}}\right]$ equal $\Delta \omega$. If the plasma is maintained at this resonant density for a suitable length of time, the resonantly driven plasma wave (with wavenumber $k_{p}$ ) will grow until it saturates due to relativistic effects. (3) This occurs when the quiver velocity of the plasma electrons in the longitudinal wave field becomes large enough that their relativistic change in mass is sufficient to void the resonance condition $\Delta \omega=\omega_{p}$. For lasers of moderate intensities $\left(\sim 10^{14} \mathrm{~W} / \mathrm{cm}^{2}\right)$, this relativistic saturation level of the longitudinal field can be at a good fraction of the maximum (sinusoidal) 1ongitudinal field possible for the given electron density, which is given as $\mathrm{eE}_{\max }=\mathrm{m} \omega_{\mathrm{p}}^{2} / \mathrm{k}_{\mathrm{p}}=\mathrm{m} \omega_{\mathrm{p}} \mathrm{c}$. This so-called cold wavebreaking field ranges from 10 to $300 \mathrm{GeV} / \mathrm{m}$ for plamas of density $10^{16}$ to $10^{19} \mathrm{~cm}^{-3}$, respectively. It is the fact that plasmas can support such high fields that makes plasma accelerators so attractve.

This paper will describe the recent experiments at UCLA on the excitation and detection of the beat-excited plasma wave as well as plans for future experiments. The paper is arranged in the following manner. The experimental apparatus will be described in Section I. Section II will cover the experimental results and interpretations while the plans for future experiments and conclusions will be given in Section III.

\section{Experimental Apparatus}

The experimental apparatus is shown schematically in Fig. 1. $\mathrm{A} \mathrm{CO}_{2}$ laser oscillator is forced to lase simultaneously on two different wavelengths $(9.6 \mu \mathrm{m}$ and $10.6 \mu \mathrm{m})$ by an intracavity absorption cell. The two-frequency, mode locked pulse train is sent through an electrooptic switch-out which selects a single, 2 nsec wide pulse for amplification. The pulse is double-passed through two amplifiers to raise the pulse energy to about $16 \mathrm{~J}$. By varying the gas pressure in the intracavity absorption cell, the ratio of the energies in the two lines can be varied. However, it is normally adjusted to give $12 \mathrm{~J}$ in the $10.6 \mu \mathrm{m}$ line and $4 \mathrm{~J}$ in the $9.6 \mu \mathrm{m}$ line. The beam is focused with an $\mathrm{f} / 8$ lens to a vacuum intensity of about $10^{13} \mathrm{~W} / \mathrm{cm}^{2}$. The plasma chamber is filled with about $1 \mathrm{~T}$ of hydrogen gas (4) which is 10cally preionized by an arc discharge a few $\mu \mathrm{sec}$ before the arrival of the $\mathrm{CO}_{2}$ pulse. The intense laser pulse rapidly heats and ionizes the gas over its approximately $8 \mathrm{~mm}$ focal depth, giving a plasma whose density is a function of gas pressure, arc voltage, and arc timing with respect to the laser pulse. Infrared light which is backscattered by the plasma via stimulated Brillouin or Raman(5) or any process is sampled off a beamsplitter and can provide information about the plasma parameters. For example, frequency analysis of the Brillouin scattered light yields information on the plasma temperature and analysis of the Raman scattered light yields electron density information. For these long $\mathrm{CO}_{2}$ pulses ( $2 \mathrm{nsec}$ ), there is quite a lot of Brillouin scattered 1ight (about 5-10\% of the incident) and a plasma shutter must be employed to protect the switch-out and oscillator optics from damage due to amplified backscatter. A portion of the incident pulse is sampled off the beamsplitter which allows a measurement of the energy and pulse shape of the two frequencies on every shot. A portion of the infrared light which is scattered in the forward direction (by the fast plasma wave) is sampled off a beamsplitter and sent through a monochrometer to be detected by a sensitive, high speed copper-doped germanium photoconductor.

The high phase velocity plasma wave is diagnosed by collective ruby-laser Thomson scattering (in

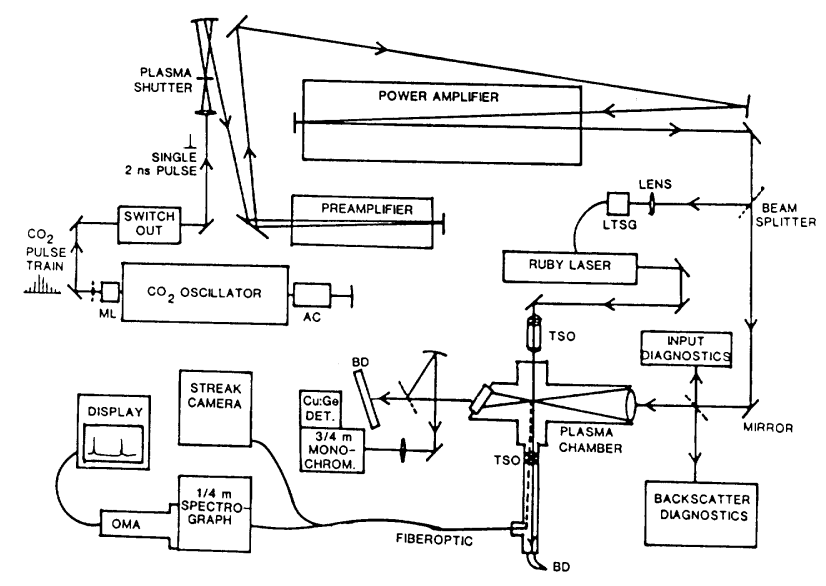

Fig. 1. Schematic diagram of the experimental arrangement. Abbreviations used are: ML, mode locker; AC, absorption cel1; LTSG, laser-tritriggered spark gap; TSO, Thomson scattering optics; BD, beam dump; OMA, optical multichannel analyzer. 
addition to the forward scattered $\mathrm{CO}_{2}$ light diagnostic). To synchronize the $20 \mathrm{nsec}$ ruby pulse to the $\mathrm{CO}_{2}$ laser pulse, a portion of the $\mathrm{CO}_{2}$ pulse is sent to a laser-triggered spark gap which fires the ruby laser at just the right moment so that the peak of the ruby pulse is at the center of the plasma chamber when the $\mathrm{CO}_{2}$ pulse arrives. The Thomson scattering optics, described elsewhere (6), cylindrically focus the ruby beam to a line-focus overlapping the $\mathrm{CO}_{2}$ focal volume. The collection optics focus the light scattered by the plasma wave onto an optical fiber which carries the signal to a spectrograph/optical multi-channel analyzer combination for frequency analysis or to a streak camera for temporal analysis. The collection end of the fiberoptic can be positioned so as to collect light at any of a range of forward scattering angles, which allows one to measure the $\mathrm{k}$ spectrum of the plasma wave. Also, the cylindrical ruby beam can be masked to illuminate various regions along the $\mathrm{CO}_{2}$ focal depth which allows one to map out the axial extent of the plasma wave.

\section{Experimental Results}

The stimulated Raman scattering instability is used to help in tuning the plasma to the resonant density. Although, as discussed below, there is some indication that the laser heated plasma has a range of densities, we can nevertheless arrange it so that the density is resonant where it is most uniform spatially. To do this, we use the fact that Raman scattering occurs where the density scalelength is longest. A product of Raman scattering is a slow phase velocity electron plasma wave which can be detected by ruby scattering at $7 \frac{1}{2}$. With the laser operating on a single frequency, this plasma wave is spontaneously excited wherever the scalelength is locally long enough to put the instability above threshold. Ruby Thomson scattering gives the frequency of this plasma wave which, if the electron temperature is known to some rough accuracy (Brillouin scattering gives $\mathrm{T}_{e}=30-50 \mathrm{eV}$ ), gives the electron

density quite accurately. In this way, the fill pressure and arc plasma conditions are established which give Raman scattering and, hence, a reasonable scalelength plasma at a density of $1.1 \times 1017 \mathrm{~cm}^{-3}$, the resonant density.

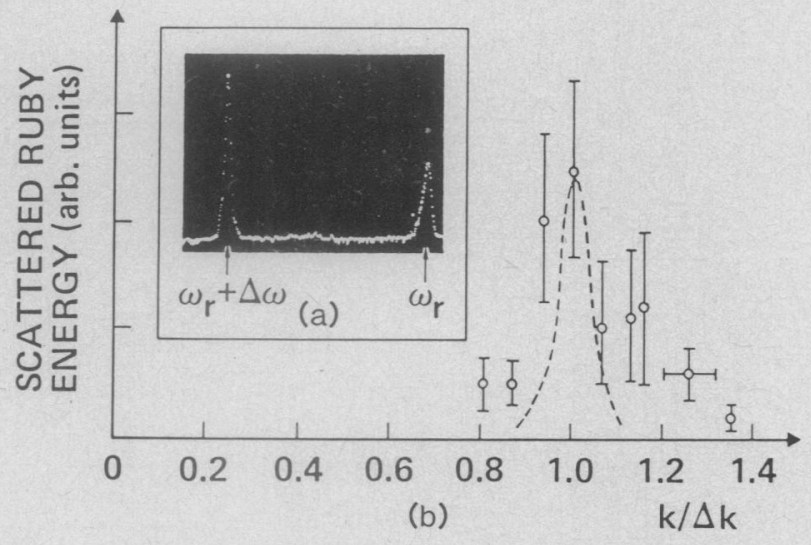

Fig. 2. (a) Time integrated frequency spectrum of the Thomson scattered light. The peak on the right is unshifted ruby (stray) light and the peak on the left is frequency shifted (scattered) 1ight. (b) The angular spectrum of the scattered light or, equivalently, the $k$ spectrum of the electron plasma wave. The dashed curve is the instrument function.
With the laser operating on two frequencies, the signal collected by the optical fiber is analyzed on the OMA (see Fig. 1). Figure 2(a) shows the typical output from the OMA. The light Thomson scattered from the fast wave is shifted from the ruby frequency by exactly $\Delta \omega$. Also, as shown in Fig. 2(b), the plasma wave wavenumber $k$ equals $\Delta k$, the wavenumber difference of the two laser lines, as it should. This verifies that the phase velocity of the plasma wave is approximately c.

A question of some importance is how nearly resonant must the density be in order to excite the wave. This is very difficult to answer experimentally with the way that the plasma is currently produced. To illustrate this, we plot the power in the forward

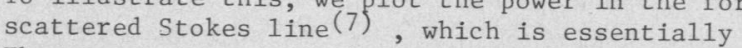
Thomson scattered $\mathrm{CO}_{2}$ light from the fast wave, vs the fill pressure. (Fig. 3) We find that there is no Stokes light until the pressure is about $1 \mathrm{~T}$. At higher pressures, the Stokes is smaller and irreproducible. Note that we expect the Stokes to peak at a total pressure of about $1 \mathrm{~T}$ since $0.7 \mathrm{~T}$ of fully ionized $\mathrm{H}_{2}$ plus $0.3 \mathrm{~T}$ of triply ionized are (essentially $\mathrm{N}_{2}$ ) produces an electron density very close to the resonant density of $1.15 \times 10^{17} \mathrm{~cm}^{-3}$. The fact that the Stokes does not return to zero beyond $1 \mathrm{~T}$ suggests that the plasma is inhomogeneous. Inhomogeneities in the $2 \mathrm{~cm}$ 1ong arc plasma are the suspected cause of the laser heated plasma nonuniformity which apparently limits the maximum length over which the density is within a few percent of resonance to

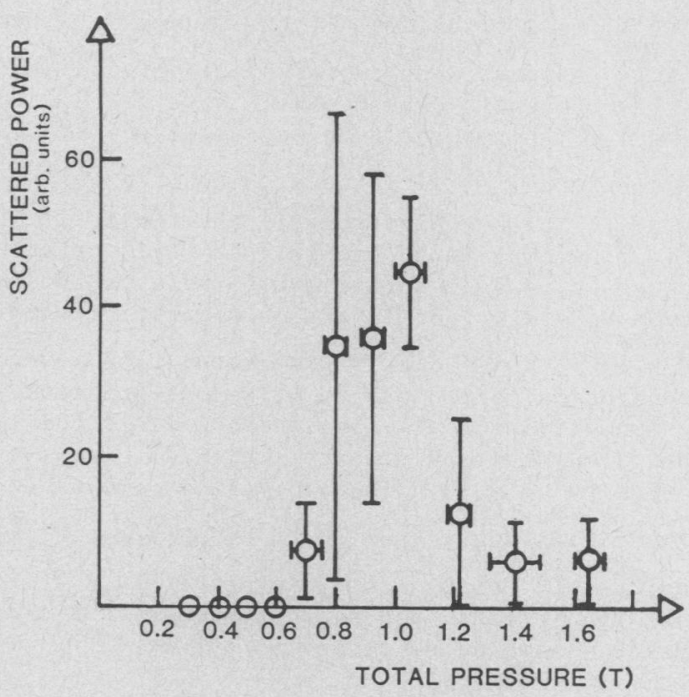

Fig. 3. Variation of the forward scattered Stokes radiation with the total fill pressure of the plasma chamber.

something like $1.8 \mathrm{~mm}$, the measured length of the fast wave. (8)

Another important, question is what limits the growth time of the fast wave. Figure $4(\mathrm{a})$ shows the pulse shape of the fast wave scattered Stokes line taken with $1 \mathrm{GHz}$ detector/scope combination (200 psec resolution). We see that growth stops after about 500 psec. Simultaneous $\mathrm{CO}_{2}$ pump transmission measurements indicate that the pump is either approximately constant or even increasing when the growth of the Stokes, and hence, the plasma wave saturates. Thus we can rule out a short pumping time as limiting 
the growth time of the plasma wave. Another possibility is that the density changes on a few hundred psec time scale. This possibility is minimized by operating at $1 \mathrm{~T}$ fill pressure so that as the plasma density builds up during ionization, it will not go through-but instead maximize at--the resonant density. Subsequent changes in the plasma density will then be governed by hydrodynamic motion of the plasma which occurs on a slow, nsec time scale. This expectation that the plasma density remains essentially constant for about a nsec is supported through the measurements of stimulated Raman scattering. Raman backscattered light was measured with the fast scope and, as shown in Fig. 4(b), was found to decay gradually after a fast, 500 psec rise. Also, time-integrated spectra of the Raman generated slow plasma waves indicate that the density was constant to within a few percent during this time. Thus, we conclude that the resonant density, once it is achieved, stays nearly resonant for on the order of $1 \mathrm{nsec}$. From these arguments, it seems quite possible that the actual temporal saturation mechanism is the expected relativistic saturation. The fact that the measured time to saturation (about 500-600 psec) agrees approximately with the idealized theoretical time to saturation (310-550 psec, depending on the assumed shape of the pump pulse) tends to confirm this conclusion. If the rate of growth of the plasma wave were to agree with theory as well, the amplitude should be $\tilde{n} / n_{0} \simeq 8 \%$. However, from the amount of Thomson scattered ruby energy, we infer the maximum amplitude of the plasma wave to be about $3 \%(8)$. This discrepency may be due to certain effects which have been neglected in the fluid theory. These include the effects of electron-ion collisions, of nonideal laser temporal and spatial coherence and perhaps most important, the effects of the presence of a large amplitude ion acoustic wave. Such an ion wave, known to be present from observations of stimulated Brillouin scattering, can damp the fast wave through the process of quasi-resonant mode coupling $(9)$ and perhaps reduce the saturation amplitude of the fast wave.

Table 1. Various design parameters for future experiments. Parameters in the first column are, in order: laser wavelengths, plasma density, plasma length, laser energy, pulse width, normalized electron quiver velocities, laser intensity, focal Rayleigh length, focal spot size. In the second column, the parameters are: relativistic $\gamma$ of the phase velocity, normalized wave amplitude, wave electric field, growth time to relativistic saturation, acceleration length, minimum injection energy, maximum energy gain, and the pump depletion length.

\section{Laser/Plasma Parameters}

\begin{tabular}{|c|c|c|c|}
\hline$\lambda_{0}, \lambda_{1}$ & $9.56,10.27 \mu \mathrm{m}$ & $\gamma_{d}$ & 14.5 \\
\hline $\mathrm{n}_{\mathrm{o}}$ & $5.8 \times 10^{16} \mathrm{~cm}^{-3}$ & $\varepsilon$ & $0.04(0.30)$ \\
\hline $\mathrm{L}_{\mathrm{p}}$ & $20 \mathrm{~cm}$ & $\mathrm{eE}$ & $1.0(6.9) \mathrm{GeV} / \mathrm{m}$ \\
\hline $\mathrm{E}_{\ell}$ & $10 \mathrm{~J}$ & $\tau_{\text {sat }}$ & $40 \mathrm{psec}$ \\
\hline${ }^{\tau} \ell$ & $50 \mathrm{psec}$ & $\mathrm{L}_{\text {acc }}$ & $1.7(1.0) \mathrm{cm}$ \\
\hline$\alpha_{0}, \alpha_{1}$ & 0.07 & $\mathrm{w}_{\text {inj }}$ & $2.4(0.3) \mathrm{MeV}$ \\
\hline$I_{0}$ & $7 \times 10^{13} \mathrm{~W} / \mathrm{cm}^{2}$ & $\Delta \mathrm{W}$ & $16(72) \mathrm{MeV}$ \\
\hline $2 z_{0}$ & $6 \mathrm{~cm}$ & $\mathrm{~L}_{\mathrm{dep}}$ & $18(0.6) \mathrm{cm}$ \\
\hline $2 \mathrm{~W}$ & $\begin{aligned} 620 \mu \mathrm{m} & \\
& \text { are va } \\
& \text { turati }\end{aligned}$ & $\approx \mathrm{Numb}$ & $\begin{array}{l}\text { n parentheses } \\
\text { tivistic sa- }\end{array}$ \\
\hline
\end{tabular}

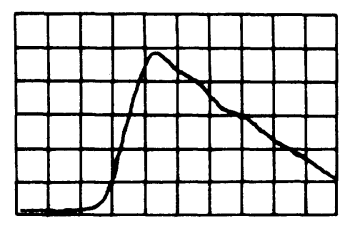

(a)



(b)
Fig. 4. Pulse shapes of (a) the forward scattered Stokes radiation and (b) Raman backscatter. Both oscilloscope traces are on $500 \mathrm{psec} / \mathrm{div}$. The dashed curve in (b) is the incident pulse shape.

\section{Future Experiments}

While experiments continue with the present apparatus (to study such issues as wave-wave coupling, etc.) the next phase of experiments are being designed. These experiments, scheduled to begin late in 1986 will basically be aimed at the controlled acceleration of injected test particles. Some limitations addressed in the last section will be minimized by making two major changes in the hardware. First, the plasma source will be a theta-pinch with the primary advantage of providing a long, preformed (fully ionized) plasma. The second change is to use a short pulse with the advantages of being "economical" with the laser energy (pulse width is matched to growth time) and of being short compared to ion-motion time scales (less Brillouin scattering). Table 1 summarizes the important parameters assuming the potential gradient will be $1 \mathrm{GeV} / \mathrm{m}$, the value already demonstrated experimentally. For comparison, the numbers in parentheses are the corresponding parameters if the wave were to be driven up to the relativistic saturation level.

\section{Acknowledgments}

We would like to thank E. M. Campbell of LLNL and P. Goldstone of LANL for the use of certain equipment. This work supported by the DOE contract DE-AT03-83ER40120, NSF grant ECS 83-10972 and the LLNL University Research Program.

\section{References}

(1) Laser Acceleration of Particles-1982, ed. by P.J. Channell, AIP Conf. Proc. No. 91 (AIP, New York).

(2) C. Joshi et al., Nature 311, 558 (1984).

(3) M. Rosenbluth and C. S. Liu, Phys. Rev. Lett. 29, 701 (1982).

(4) Actually, $0.3 \mathrm{~T}$ of air is included to aid in ionization.

(5) D. W. Forslund et al., Phys. Fluids 18, 1002 (1975).

(6) C. E. Clayton et al., (submitted to App1. Opt.).

(7) Two frequency downshifted (Stokes and secondStokes) and one upshifted (anti-Stokes) sidebands are observed in the forward direction.

(8) C. E. Clayton et al., (accepted by Phys. Rev. Lett.).

(9) P. K. Kaw et a1., Phys. Fluids 16, 1967 (1973). 